Research Paper

\title{
Combination of PD-L1 expression and NLR as prognostic marker in patients with surgically resected non-small cell lung cancer
}

\author{
Xinyue Wang1,2,3,4, Lianjing Cao5, Shouying Li1,2,3,4, Fan Wang1,2,3,4, Dingzhi Huang1,2,3,4, Richeng Jiang1,2,3,4 \\ 1. Tianjin Medical University Cancer Institute \& Hospital, National Clinical Research Center for Cancer \\ 2. Key Laboratory of Cancer Prevention and Therapy, Tianjin \\ 3. Tianjin's Clinical Research Center for Cancer \\ 4. Department of Thoracic Oncology, Tianjin Lung Cancer Center, Tianjin Cancer Institute \& Hospital, Tianjin Medical University, Tianjin 300060, PR China \\ 5. State Key Laboratory of Oncology in South China, Collaborative Innovation Center for Cancer Medicine, Sun Yat-sen University Cancer Center, Guangzhou \\ 510060, China
}

$\triangle$ Corresponding author: Richeng Jiang, Postal Address: Department of Thoracic Oncology, Tianjin Cancer Institute \& Hospital, Huan-Hu-Xi Road, Hexi district, Tianjin, China. Phone: 86-22-23340123-3200, Fax: 86-22-23537796. E-mail: jiangricheng@tjmuch.com

(c) The author(s). This is an open access article distributed under the terms of the Creative Commons Attribution License (https://creativecommons.org/licenses/by/4.0/). See http://ivyspring.com/terms for full terms and conditions.

Received: 2019.02.27; Accepted: 2019.08.29; Published: 2019.10.22

\begin{abstract}
Background: In recent years, great improvement has been made in immunotherapies for non-small cell lung cancer (NSCLC). Current data have suggested that Programmed cell death ligand 1 (PD-L1) expression might not be an ideal marker for patient selection in isolation. Evidence has been increasing that alternative markers, such as neutrophil-to-lymphocyte ratio (NLR), a biomarker of systemic inflammation response (SIR) previously associated with outcomes in a variety of cancers including NSCLC, might be a predictor for patient selection and the response to therapy. No reports have examined the prognostic value of combination of PD-Ll expression and inflammatory markers such as NLR in NSCLC. This retrospective study explores the relationship between NLR and PD-L1 expression in NSCLC as well as the prognostic value of combination of PD-LI expression and NLR.

Method: We evaluated tumor PD-L1 expression in 235 surgically resected NSCLC cases by immunohistochemical analysis. Carcinoma cells showing membranous staining for PD-L1 were considered PD-L1-positive cells (Figure 1). Cases with $\geq 1 \%$ tumor membrane staining were considered PD-LI-positive. The association of clinicopathological characteristics with PD-L1 expression was assessed by univariate and multivariate analyses. Moreover, univariate and multivariate analyses were performed to evaluate the predictive impact of PD-LI expression and other factors on disease-free survival (DFS) and overall survival (OS).

Result: PD-LI protein expression was elevated in $34.0 \%$ of patients at cut-off value of $1 \%$. Univariate analyses showed that PD-L1 expression was significantly higher in men $\left(X^{2}=5.226, P=0.030\right)$, heavy smokers $\left(X^{2}=18.650, P<0.001\right)$, and patients with squamous cell carcinoma $\left(X^{2}=4.036, P=0.045\right)$. No correlations were noted between PD-L1 expression and age, EGFR mutation status or clinical stage. No significant correlations between PD-L1 protein expression and NLR were found. Multivariate logistic regression revealed that smoking index $\geq 400$ was independent predictor of PD-L1 expression (odds ratio [OR], 3.375; $P<0.001$ ). The results of univariate survival analyses showed that clinical stage (log-rank $\left.X^{2}=7.876, P=0.019\right)$ was associated with DFS. Smoking index (log-rank $\left.X^{2}=4.832, P=0.028\right)$, clinical stage (log-rank $\left.X^{2}=7.582, P=0.023\right)$ and adjuvant treatment (log-rank $\left.X^{2}=5.440, P=0.020\right)$ were significantly associated with OS. Neither PD-LI expression nor NLR was found to be associated with DFS or OS. Of interest, when patients were divided in two groups according to combined PD-L1/NLR: patients with PD-L1+/ high NLR as Group 1, other patients as Group 2, Group 1 had significantly shorter DFS as well as OS than Group 2 (DFS: log-rank $X^{2}=5.231$, $P=0.022$, Figure $2 A$; OS: log-rank $X^{2}=4.742, P=0.029$, Figure $2 B$ ). In the multivariate analysis, Cox proportional hazards regression models showed that, PD-LI+/ high NLR was associated with a significantly shorter DFS and OS (hazard ratio $[H R], 1.394, P=0.040 ; H R, 1.442, P=0.042$, respectively). Stratified analysis showed that the prognostic value of combined PD-L1/NLR can only be observed in cases without epidermal growth factor receptor (EGFR) mutations (DFS: log-rank $X^{2}=5.593, P=0.018$, Figure 2C, OS: log-rank $X^{2}=9.323, P=0.002$, Figure $2 D$ ). In EGFR mutation subgroup, combination of PD-L1 expression and NLR has no relationship with DFS or OS.
\end{abstract}

Conclusion: We found that combination of PD-L1 expression and NLR may be a promising prognostic indicator, and may also be a good marker for tumor recurrence, especially in the patients with wild-type EGFR.

Key words: neutrophil-lymphocyte ratio, programmed death ligand-1, immunohistochemical analysis, prognostic marker, non-small cell lung cancer 


\section{Introduction}

Lung cancer is currently the top cause of cancer deaths worldwide [1]. Non-small cell lung cancer (NSCLC), which accounts for more than $80 \%$ of all lung cancers, usually presents in a late stage in approximately $80 \%$ of cases. Despite rapid progress in the diagnosis and treatment of lung cancer, its prognosis remains poor, with 5-year survival rates of less than $15 \%$. In recent years, great improvement has been made in immunotherapies for NSCLC, particularly monoclonal antibodies targeting the cytotoxic T-lymphocyte-associated protein 4 (CTLA-4), programmed death-1(PD-1) and its ligand (PD-L1). Although immune checkpoint inhibitors appear capable of producing durable responses compared to existing treatments, unfortunately a substantial proportion of patients treated with immune checkpoint inhibitors do not respond[2,3].

Accordingly, the identification of biomarkers that predict the clinical efficacy of immune checkpoint blockade therapy is urgent. Furthermore, effective combined therapies with immunotherapy are required for improved clinical benefit. Current data have indicated that the correlation between PD-L1 expression by immunohistochemistry and the response to immune checkpoint inhibition varies by both tumors histologic features, suggesting that PD-L1 might not be an ideal marker for patient selection in isolation [4]. Nonetheless, evidence has been increasing that alternative markers, such as neutrophil-to-lymphocyte ratio (NLR), a biomarker of systemic inflammation response (SIR) previously associated with outcomes in a variety of cancers including NSCLC[5-12], might be a predictor for patient selection and the response to therapy. High NLR has been reported to be associated with poor prognosis in patients with advanced NSCLC that were receiving immunotherapies[13, 14]. However, no reports have examined the potential prognostic value of combination of PD-L1 expression and inflammatory markers such as NLR. Herein, this retrospective study explores the relationship between NLR and PD-L1 expression in NSCLC as well as the prognostic value of combination of PD-L1 expression and NLR. Elucidation of the clinical significance of PD-L1 protein expression in combine with inflammatory marker in NSCLC may provide insights into patient selection and effective strategies for PD-1/PD-L1 inhibitory treatment.

\section{Material \& Methods}

\section{Patients}

Patients who underwent surgery for primary adenocarcinoma (AD) and squamous cell carcinoma (SCC) between 2012 and 2015 were identified from a retrospective review of a prospectively maintained database at Tianjin Cancer Institute \& Hospital, Tianjin Medical University, Tianjin P.R. China. We excluded patients: (1) who were lost to follow up, (2) without R0 resections, (3) with distant metastases, (4) had insufficient laboratory data, (5) had clinical evidence of infection or other inflammatory conditions, (6) had received preoperative chemotherapy or irradiation, (7) whose matched paraffin embedded formalin-fixed (FFPE) tissue specimens were unavailable. Finally, 235 patients entered our study. Information about demographics, data regarding surgical procedures, preoperative blood variables, postoperative course, pathologic findings, and follow-up was collected. The NLR was defined as the absolute neutrophil count divided by the absolute lymphocyte count. DFS and OS were stratified by median of NLR (2.3), which was also consisting with previous studies [15-18] . Epidermal growth factor receptor (EGFR) mutation status was detected by real-time PCR or DNA sequencing as previously described [19].

All Patients were observed until death or July 1, 2017. The median follow-up period was 35 months. Disease-free survival (DFS) was defined as the time from resection to the first disease recurrence and was censored at the last follow-up date if no events had occurred. Overall survival (OS) was calculated from the date of surgery to the date of death or last follow-up. Prior consent from all patients and approval from the Research Ethics Committee of Tianjin University were obtained for the use of clinical and pathological data.

\section{Immunohistochemistry for PD-L1}

Tumor PD-L1 expression was evaluated by immunohistochemistry on formalin-fixed and paraffin embedded tumor tissue sections according to the previously described PD-L1 immunohistochemistry protocol [19]. Briefly, formalin-fixed tissue sections were dewaxed with xylene followed by rehydrated through a graded series of ethanol and washed in distilled water $\left(\mathrm{dH}_{2} \mathrm{O}\right)$. Antigen retrieval was performed with EDTA buffer per the manufacture's, then sections were incubated in 3\% hydrogen peroxide $\left(\mathrm{H}_{2} \mathrm{O}_{2}\right)$ for $10 \mathrm{~min}$ in order to inhibit endogenous peroxidase activity. The sections were incubated overnight at $4^{\circ} \mathrm{C}$ with the monoclonal antibodies at $4^{\circ} \mathrm{C}$ overnight. To visualize 
the antigen, the immune complex was detected with a DAKO EnVision Detection System (Dako). Finally, sections were then counterstained with hematoxylin, and mounted. The primary antibody was an anti-human PD-L1 rabbit monoclonal antibody (rabbit anti-PD-L1 $\mathrm{XP}^{\circledR} \mathrm{mAb}$ 1:100, E1L3N, cell signaling Technology, Danvers, MA, United States of America (USA)). Carcinoma cells showing membranous staining for PD-L1 were considered PD-L1-positive cells. The proportion of PD-L1-positive cells was independently estimated as the percentage of total carcinoma cells in whole sections by two investigators (X.W. and L.C.). Cases with $\geq 1 \%$ tumor membrane staining were considered PD-L1-positive.

\section{Statistical Analysis}

Categorical variables are presented as numbers and percentages. Distribution of continuous variables is reported as median and range. Qualitative data were compared by the $\chi^{2}$ test or Fisher's exact test when necessary. The logistic regression model was used to estimate influence of preoperative factors on PD-L1 expression. Survival probability was estimated by the Kaplan-Meier method, and log-rank test was used for the comparison of survival. Multivariate analyses were performed using the Cox proportional hazards regression model to evaluate significant recurrence predictors and prognostic factors. All tests were 2-sided. Statistical analyses were performed in SPSS 17.0 for Windows software (SPSS Inc). $P$ values of $<0.05$ were considered to indicate statistical significance.

\section{Results}

\section{Association between PD-Ll expression and clinicopathological characteristics}

The clinicopathological characteristics of the 235 patients with NSCLC (130 with AD and 105 with SCC) are summarized in Table 1 . The median age was 59 years (range 32-78). One hundred and fifty-three $(65.1 \%)$ patients were male and $93(39.6 \%)$ were heavy smokers (smoking index $\geq 400$ ). Tumors of stages I, II, and III were observed in 112 (47.7\%), 45 (19.1\%) and $78(33.2 \%)$ cases, respectively. Post-operative therapy was performed in 94 patients: 88 patients received chemotherapy; 3 were exposed to EGFR-TKI targeted therapy, 3 received radiation therapy, and 5 received both chemotherapy and radiotherapy. EGFR-mutation status was present in 48 patients (20.4\%) and the other 187 (79.6\%) cases had EGFR wild-type tumors.

Immunohistochemical staining for PD-L1 was detected at the membrane of tumor cells (Figure 1). Eighty $(34.0 \%)$ patients were positive for PD-L1 at the
$1 \%$ cut-off value. The associations between PD-L1 expression and the clinicopathological features of the patients are summarized in Table 1. PD-L1 expression was significantly higher in men, heavy smokers, and patients with squamous cell carcinoma. No correlations were noted between PD-L1 expression and age, EGFR mutation status or clinical stage. Eighty $(34.0 \%)$ patients had an $N L R \geq 2.3$. No significant correlations between PD-L1 protein expression and NLR were found. In a multivariable logistic regression analysis, smoking index $\geq 400$ (odds ratio [OR], 3.375; 95\% CI, 1.922-5.926; $P<0.001$ ), was found to be independently associated with PD-L1 expression.

Table 1. Correlation between PD-L1 expression and clinicopathological characteristics

\begin{tabular}{|c|c|c|c|c|c|}
\hline \multirow{2}{*}{$\begin{array}{l}\text { Variables } \\
\text { Age (years) }\end{array}$} & \multirow[t]{2}{*}{$\mathrm{N}$} & \multirow[t]{2}{*}{$\chi^{2}$} & \multicolumn{3}{|c|}{$\begin{array}{l}\text { PD-L1 expression }(\%) \\
\text { Negative Positive } P \text {-value }\end{array}$} \\
\hline & & & & & \\
\hline$<60$ & 124 & 1.349 & $86(69.3)$ & $38(30.6)$ & 0.245 \\
\hline$\geq 60$ & 111 & & $69(62.2)$ & $42(37.8)$ & \\
\hline \multicolumn{6}{|l|}{ Gender } \\
\hline Male & 153 & 5.226 & $93(60.8)$ & $60(39.2)$ & 0.030 \\
\hline Female & 82 & & $62(75.6)$ & $20(24.4)$ & \\
\hline \multicolumn{6}{|c|}{ Smoking Index } \\
\hline$<400$ & 142 & 18.650 & $109(76.8)$ & $33(23.2)$ & $<0.001$ \\
\hline$\geq 400$ & 93 & & $46(49.5)$ & $47(50.5)$ & \\
\hline \multicolumn{6}{|c|}{ Clinical stage } \\
\hline I & 112 & 5.097 & $82(73.2)$ & $30(26.8)$ & 0.078 \\
\hline II & 45 & & $26(57.8)$ & $19(42.2)$ & \\
\hline IIIA & 78 & & $47(60.3)$ & $31(39.7)$ & \\
\hline \multicolumn{6}{|l|}{ Histology } \\
\hline $\mathrm{AD}$ & 130 & 4.036 & $93(71.5)$ & $37(28.5)$ & 0.045 \\
\hline SCC & 105 & & $62(59.0)$ & $43(41.0)$ & \\
\hline \multicolumn{6}{|c|}{ EGFR mutation } \\
\hline No & 187 & 0.210 & $122(65.2)$ & $65(34.8)$ & 0.647 \\
\hline Yes & 48 & & $33(68.8)$ & $15(31.3)$ & \\
\hline \multicolumn{6}{|l|}{ NLR } \\
\hline$<2.3$ & 155 & 0.005 & $102(65.8)$ & $53(34.2)$ & 0.946 \\
\hline$\geq 2.3$ & 80 & & $53(66.3)$ & $27(33.8)$ & \\
\hline
\end{tabular}

SCC: squamous cell carcinoma. AD: adenocarcinoma.

Smoking index $=($ number of cigarettes per day $) \times($ duration in years $)$ $P$-values $<0.05$ in bold

\section{Univariate and Multivariate Survival Analyses in All Patients}

The median follow-up period was 36.9 months. During the observation period, $132(56.2 \%)$ patients died. Univariate and multivariate analyses were performed to evaluate the predictive impact of PD-L1 expression and other clinicopathological factors on OS and DFS (Table 2). The results of univariate analyses showed that clinical stage (log-rank $X^{2}=7.876$, $P=0.019)$ was associated with DFS. Smoking index (log-rank $X^{2}=4.832, P=0.028$ ), clinical stage (log-rank $X^{2}=7.582, P=0.023$ ) and adjuvant treatment (log-rank $\chi^{2}=5.440, P=0.020$ ) were significantly associated with OS. Neither PD-L1 expression nor NLR was found to be associated with DFS or OS. Of interest, when 
patients were divided in two groups according to combined PD-L1/NLR: patients with PD-L1+/ high NLR as Group 1, other patients as Group 2, DFS and OS showed significant difference between groups.
Group 1 had significantly shorter DFS as well as OS than Group 2 (DFS: log-rank $X^{2}=5.231, P=0.022$, Figure 2A; OS: $\log$-rank $\chi^{2}=4.742, P=0.029$, Figure 2B).
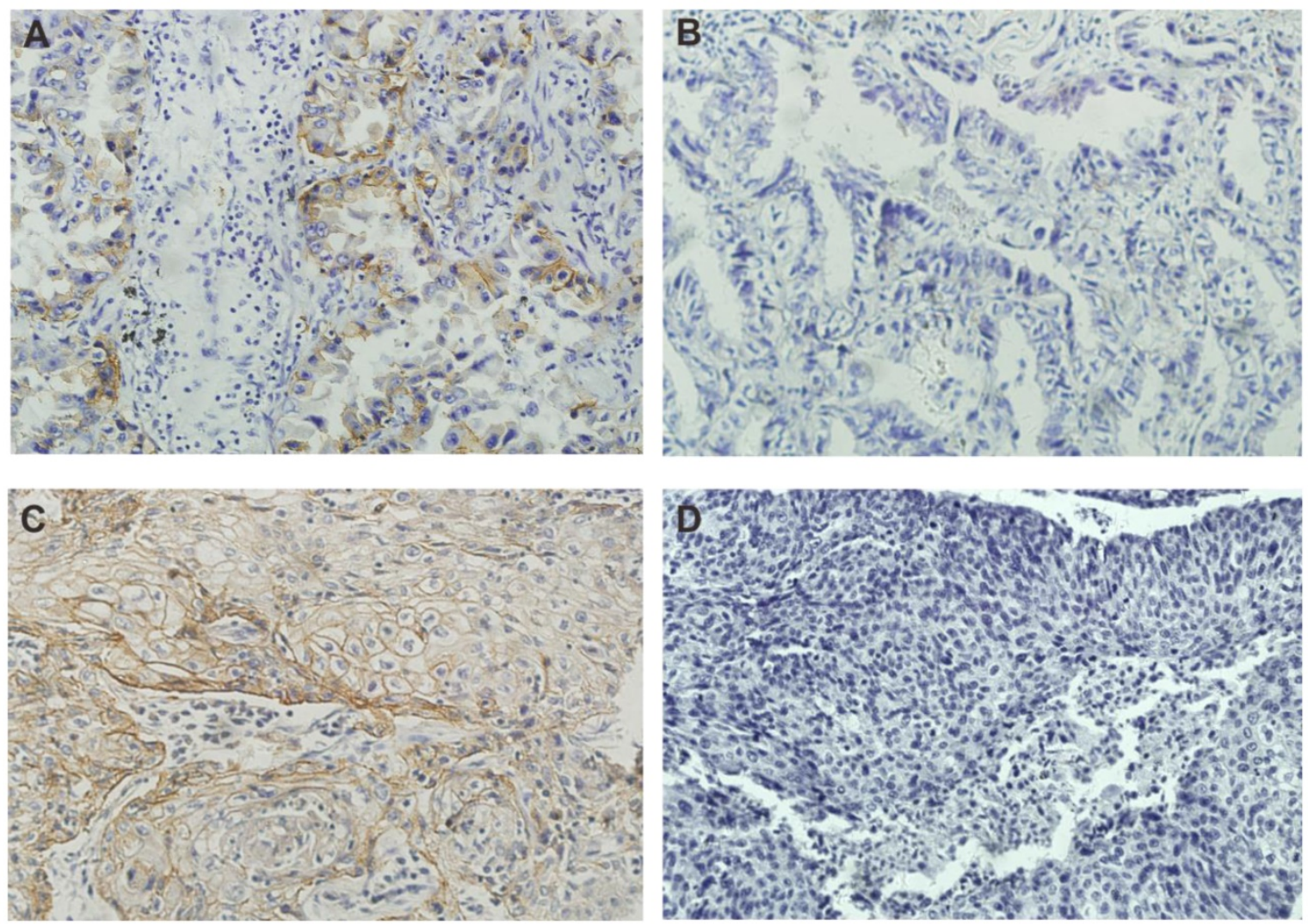

Figure 1. Expression of PD-L1 in lung adenocarcinomas (A, B) and squamous cell carcinomas (C, D). Representative images of PD-L1 positive expression (A, C) and negative expression (B, D). Magnification $\times 200$.
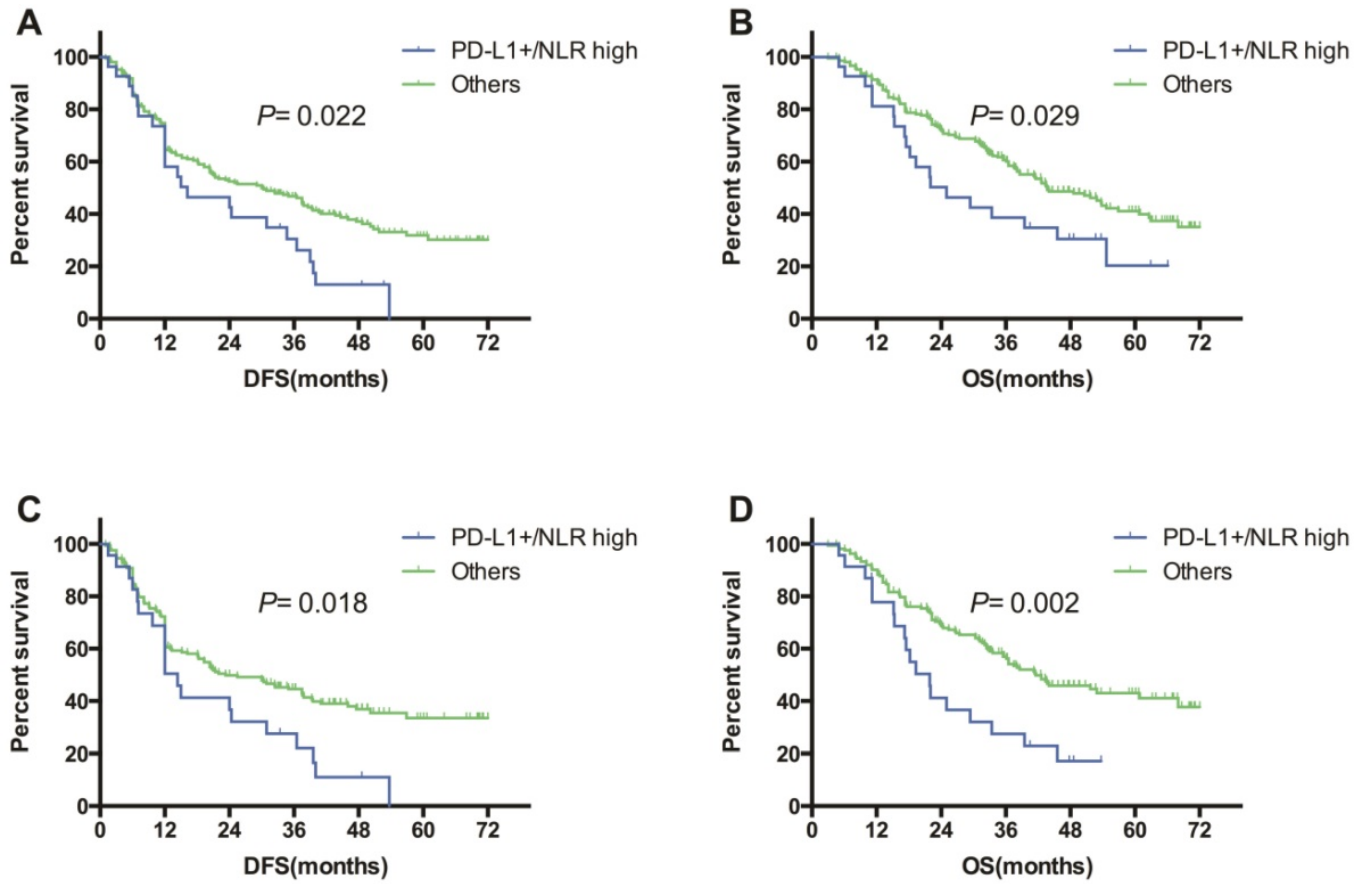

Figure 2. Kaplan-Meier survival curves of DFS (A, C) and OS (B, D) based on combination of PD-L1 expression and NLR (PD-L1+/ NLR high vs. others) in all patients (A, B), and in patients with wild-type $\operatorname{EGFR}(\mathrm{C}, \mathrm{D})$. 
Table 2. Predictive and prognostic values of variables by univariate analysis

\begin{tabular}{|c|c|c|c|c|c|c|c|}
\hline Variables & $\mathrm{N}$ & DFS & & & OS & & \\
\hline & & $\begin{array}{l}\text { Median } \\
\text { DFS }\end{array}$ & $\chi^{2}$ & $P$-value & $\begin{array}{l}\text { Median } \\
\text { OS }\end{array}$ & $\chi^{2}$ & $P$-value \\
\hline \multicolumn{8}{|l|}{ All patients } \\
\hline \multicolumn{8}{|l|}{ Age (years) } \\
\hline$<60$ & 124 & 32.4 & 0.680 & 0.410 & 43.6 & 0.923 & 0.337 \\
\hline$\geq 60$ & 111 & 21.0 & & & 42.6 & & \\
\hline \multicolumn{8}{|l|}{ Gender } \\
\hline Male & 153 & 32.4 & 0.463 & 0.496 & 44.0 & 0.001 & 0.971 \\
\hline Female & 82 & 23.9 & & & 38.6 & & \\
\hline \multicolumn{8}{|c|}{ Smoking Index } \\
\hline$<400$ & 142 & 30.9 & 1.153 & 0.283 & 51.7 & 4.832 & 0.028 \\
\hline$\geq 400$ & 93 & 20.7 & & & 35.5 & & \\
\hline \multicolumn{8}{|l|}{ Clinical stage } \\
\hline I & 112 & 37.4 & 7.876 & 0.019 & 54.7 & 7.582 & 0.023 \\
\hline II & 45 & 32.4 & & & 41.2 & & \\
\hline IIIA & 78 & 16.2 & & & 35.5 & & \\
\hline \multicolumn{8}{|l|}{ Histology } \\
\hline $\mathrm{AD}$ & 130 & 25.5 & 1.045 & 0.307 & 43.7 & 0.130 & 0.719 \\
\hline SCC & 105 & 32.4 & & & 41.2 & & \\
\hline \multicolumn{8}{|c|}{ Adjuvant treatment } \\
\hline No & 147 & 21.0 & 0.371 & 0.543 & 36.5 & 5.440 & 0.020 \\
\hline Yes & 88 & 36.5 & & & 52.9 & & \\
\hline \multicolumn{8}{|c|}{ EGFR mutation } \\
\hline No & 187 & 21.3 & 0.730 & 0.393 & 38.1 & 2.798 & 0.094 \\
\hline Yes & 48 & 37.5 & & & 54.7 & & \\
\hline \multicolumn{8}{|l|}{ PD-L1 } \\
\hline Negative & 155 & 30.0 & 1.166 & 0.280 & 43.4 & 0.667 & 0.414 \\
\hline Positive & 80 & 24.0 & & & 41.2 & & \\
\hline \multicolumn{8}{|l|}{ NLR } \\
\hline$<2.3$ & 155 & 35.0 & 3.366 & 0.067 & 44.0 & 1.624 & 0.202 \\
\hline$\geq 2.3$ & 80 & 20.4 & & & 38.1 & & \\
\hline \multicolumn{8}{|c|}{ PD-L1 and NLR } \\
\hline PD-L1+/NLR & 27 & 16.2 & 5.231 & 0.022 & 24.9 & 4.742 & 0.029 \\
\hline \multicolumn{8}{|l|}{ high } \\
\hline Others & 208 & 30.0 & & & 43.7 & & \\
\hline \multicolumn{8}{|c|}{ Patients with EGFR wild type } \\
\hline PD-L1+/NLR & 23 & 14.4 & 5.593 & 0.018 & 21.9 & 9.323 & 0.002 \\
\hline \multicolumn{8}{|l|}{ high } \\
\hline Others & 164 & 23.3 & & & 41.6 & & \\
\hline \multicolumn{8}{|c|}{ Patients with EGFR mutation } \\
\hline PD-L1+/NLR & 4 & 34.7 & 0.003 & 0.959 & 62.3 & 1.794 & 0.180 \\
\hline \multicolumn{8}{|l|}{ high } \\
\hline Others & 44 & 38.6 & & & 53.8 & & \\
\hline \multicolumn{8}{|c|}{ Patients with adjuvant chemotherapy } \\
\hline PD-L1+/NLR & 9 & 24.4 & 4.582 & 0.032 & 45.6 & 0.120 & 0.729 \\
\hline \multicolumn{8}{|l|}{ high } \\
\hline Others & 79 & 37.5 & & & 52.9 & & \\
\hline \multicolumn{8}{|c|}{ Patients without adjuvant chemotherapy } \\
\hline PD-L1+/NLR & 18 & 12.0 & 1.688 & 0.194 & 19.3 & 5.368 & 0.021 \\
\hline \multicolumn{8}{|l|}{ high } \\
\hline Others & 129 & 23.3 & & & 38.6 & & \\
\hline
\end{tabular}

SCC: squamous cell carcinoma. AD: adenocarcinoma.

Smoking index $=($ number of cigarettes per day $) \times($ duration in years $)$.

$P$-values $<0.05$ in bold.

In the multivariate analysis, Cox proportional hazards regression models showed that stage III and PD-L1+/ high NLR were associated with a significantly shorter DFS (stage III vs. stage I-II, HR, 1.559, $P=0.009$; Group 1 vs. Group 2: HR, 1.394, $P=0.040$, respectively). Moreover, clinical stage, smoking index, adjuvant treatment, and combined PD-L1/NLR remained independent factors of OS (stage III vs. stage I-II, HR, 1.831, $P=0.001 ; \mathrm{SI} \geq 400$ vs.
$\mathrm{SI}<400, \mathrm{HR}, 1.616, P=0.008$; with vs. without adjuvant treatment, HR, 0.544, $P=0.001$; Group 1 vs. Group 2: HR, 1.442, $P=0.042$, respectively, Table 3).

Table 3. Predictive and prognostic values of variables by multivariate analysis

\begin{tabular}{|c|c|c|c|c|c|}
\hline Variables & $\mathrm{N}$ & DFS & & OS & \\
\hline & & HR $(95 \% \mathrm{CI})$ & $P$-value & $\mathrm{HR}(95 \% \mathrm{CI})$ & $P$-value \\
\hline \multicolumn{6}{|l|}{ Clinical stage } \\
\hline IIIA & 78 & $1.559(1.123-2.166)$ & 0.009 & $1.831(1.2749-2.632)$ & 0.001 \\
\hline I- II & 157 & & & & \\
\hline \multicolumn{6}{|c|}{ Smoking Index } \\
\hline$\geq 400$ & 93 & & & $1.616(1.136-2.300)$ & 0.008 \\
\hline$<400$ & 142 & & & & \\
\hline \multicolumn{6}{|c|}{ Adjuvant treatment } \\
\hline Yes & 88 & & & $0.544(0.376-0.788)$ & 0.001 \\
\hline No & 147 & & & & \\
\hline \multicolumn{6}{|c|}{ PD-L1 and NLR } \\
\hline $\begin{array}{l}\text { PD-L1+/NLR } \\
\text { high }\end{array}$ & 27 & $1.394(1.071-1.811)$ & 0.040 & $1.442(1.064-1.954)$ & 0.042 \\
\hline Others & 208 & & & & \\
\hline
\end{tabular}

\section{DFS and OS According to NLR/PD-L1 in Subgroups}

Subgroup analyses were performed for EGFR mutation status and adjuvant treatment (Table 2). In EGFR mutation subgroup, the results of wild-type subgroup analysis were similar with the results of entire cohort analysis that mentioned above: neither PD-L1 expression nor NLR was found to be associated with DFS or OS, PD-L1+ / high NLR was found to be associated with worse DFS (log-rank $\chi^{2}=5.593, P=$ 0.018 , Figure 2C) and OS (log-rank $\chi^{2}=9.323, P=0.002$, Figure 2D). However, in EGFR mutation subgroup, combination of PD-L1 expression and NLR has no relationship with DFS or OS. In addition, when patients were stratified according to adjuvant therapy, PD-L1+/ high NLR was associated with inferior OS (log-rank $X^{2}=5.386, P=0.021$ ) and a trend for worse DFS (log-rank $\left.\chi^{2}=1.688, P=0.194\right)$ in patients without adjuvant chemotherapies.

In patients received adjuvant chemotherapies,

PD-L1+/ high NLR was identified to be associated with worse DFS (log-rank $\chi^{2}=4.582, P=0.032$ ) but not OS $\left(\log\right.$-rank $\left.\chi^{2}=0.120, P=0.729\right)$.

\section{Discussion}

In the present study, PD-L1 protein expression was elevated in $34.0 \%$ of patients with NSCLC who underwent surgery (41.0\% of lung SCC samples and $28.5 \%$ of lung AD samples). PD-L1-positive expression was more frequently observed in male, heavy smokers and patients with SCC. Multivariate analysis revealed that smoking index $\geq 400$ was independent predictor of PD-L1 expression. No 
significant correlations between PD-L1 protein expression and NLR or EGFR mutation status had been found. Patients with pretreatment NLR $>2.3$ and PD-L1 expression was associated with inferior DFS and OS. Moreover, we demonstrated that such poor prognosis was only observable in cases without EGFR mutations, and the prognostic effect for NLR/PD-L1 might be affected by adjuvant therapy and subsequent treatment.

In previous reports, clinical factors, such as smoking history, were reported to be associated with the PD-L1 expression. Wu et al. reported that PD-L1 protein expression is higher in men than women, smokers than never smokers [20]. Takada et al. demonstrated that PD-L1 positivity was significantly associated with male sex, smoking and squamous cell carcinoma [21].Another study showed the high-PD-L1-expression group had a significantly higher proportion of smokers compared with the low-expression group [22]. These data are consistent with the results of our study.

Previous researches have also shown that PD-L1 protein expression is associated with EGFR mutations. Takada et al. pointed out that PD-L1 positivity was significantly associated with wild-type EGFR [23]. On the contrary, other studies showed that PD-L1 expression was significantly associated with the presence of EGFR mutations [24, 25], which was not observed in our study. The reasons for these discrepancies may be due to the admixture of $\mathrm{AD}$ and SCC in our analysis as well as different antibodies and cutoffs. Although our study have found no significant correlations between PD-L1 protein expression and EGFR mutation status, our stratified analysis showed that the prognosis value of combined PD-L1/NLR could only be observed in wild-type patients but not EGFR-mutated patients.

Many studies have evaluated the prognostic impact of PD-L1 protein expression in NSCLC, including the present study. However, the results of these studies vary: some studies have shown that expression of PD-L1 was correlated with poor clinical outcomes in NSCLC[26, 27]; while others showed a favorable prognosis for NSCLC with PD-L1 expression[28, 29].In our study, however, no significant correlations between PD-L1 protein expression and prognosis were found, when we analyzed the survival in strata of histology, still no association was found between PD-L1 protein expression and DFS or OS (data not shown). As described above, different antibodies and cutoffs may account for some of the discrepancies.

There are a number of well-established systemic inflammation-based prognostic scores for patients with NSCLC [9]. NLR, which reflects alterations in peripheral blood leukocytes associated with systemic inflammatory response, has been studied extensively as a marker of tumor outcomes [5-8, 10-12]. Moreover, in the era of immunotherapy, studies have shown that systemic inflammation markers are associated with the outcome of NSCLC patients that treated with nivolumab. Elevated baseline NLR has also been associated with worse outcomes in patients with NSCLC treated with nivolumab[13]. Data from the Swiss has also suggested that pre-treatment NLR was associated with shorter OS and PFS and with lower response rate in patients with metastatic NSCLC treated with nivolumab[14].Our analyses revealed that, patients with pretreatment NLR>2.3 and PD-L1 expression had significantly shorter DFS and OS, suggesting a possible benefit population with immune checkpoint inhibitors. Further studies are needed to determine whether combination of PD-L1 expression and NLR are predictive or prognostic in NSCLC patients treated with immune checkpoint inhibitors.

Mechanism of regulation of PD-L1 expression has not yet been sufficiently clarified. Previous reports often focused on oncogene-driven mechanisms $[30,31]$, while a recent study indicated that a distinct, inducible mechanism was operative for PD-L1 expression. Specifically, IFN- $\gamma$ secreted by TILs promotes PD-L1 expression by tumors and other cells in the immediate tumor microenvironment, which in turn leads to dysregulation of T-cell effector functions via inhibitory PD-1 interaction [32]. Inflammation-induced PD-L1 expression by IFN- $\gamma$ differs from oncogene-induced PD-L1 expression in that PD-L1 expression depends on the time and site of the immune response. We therefore investigated the association between PD-L1 expression and NLR, and whether or not combination of PD-L1 expression and NLR in resectable NSCLC patients can be a prognostic indicator. Our result showed that patients with PD-L1expression and high NLR tend to show poor outcomes. Therefore, we could since speculate that in cases with poor outcomes included those that both raised NLR and broad PD-L1 expression, inflammation-induced PD-L1 expression may be dominant, and an immunosuppressive state in relation to over-production of neutrophils may have occurred, and at the same time, PD-1-mediated tumor immune escape by which the cancer cells can become progressive may have also been activated.

The validation of PD-L1 expression and inflammatory markers may be significant because they offer the potential for a feasible test that can be used to help evaluate the patient's prognosis. However, the tumor-host immune and inflammatory response is a complex interaction, with the key processes 
underlying this response still unclear. Our study has several limitations. First, the current study included patients with only operable NSCLC, and these patients were not treated with PD-1 or PD-L1 axis therapies. Further studies in patients treated with PD-1 axis therapies should be performed to confirm the findings obtained in the current study. Second, the PD-L1 analysis for surgically resected NSCLC was conducted using a specific antibody against PD-L1 (E1L3N) and with one cut-off value. Immunohistochemical staining of PD-L1 had been performed using different PD-L1 antibody clones in the different studies and clinical trials[33-37], the distribution of PD-L1 expression at the $1 \%$ and $50 \%$ cut points closely reflected the percentages of the population considered positive in the Keynote[34] and CheckMate[35] studies. Many studies compare the commercially available clones. It was shown that the antibodies, from the perspective of interaction with the PD-L1 epitope, are most likely only subtly different [38-42]. PD-L1 staining using clone E1L3N has been shown to render equivalent results to antibodies 22C3 and 28-8 in previous studies [38, 43]. Parra et al [40] compared a large number of different PD-L1 commercial clones, showed these antibody clones are comparable and presented the feasibility of an equally high sensitivity of PD-L1 staining using clone E1L3N. Therefore, we consider our PD-L1 staining using E1L3N as valid and reliable.

\section{Conclusion}

Taken together, our findings indicate that combination of PD-L1 expression and NLR may be a promising prognostic indicator, and may also be a good marker for tumor recurrence. However, to suggest potential intervention strategies aimed at cancer-associated inflammation so as to improve outcome of immune checkpoint blockade, further study of these mechanisms, including research from a molecular biological approach, and covering more advanced NSCLC cases, is required.

\section{Acknowledgments}

This work was supported by grants from the Tianjin Municipal Science and Technology Commission Key Application Research Projects (No. 11JCZDJC18900) for Richeng Jiang.

\section{Competing Interests}

The authors have declared that no competing interest exists.

\section{References}

1. Bray F, Ferlay J, Soerjomataram I, Siegel RL, Torre LA, Jemal A. Global cancer statistics 2018: GLOBOCAN estimates of incidence and mortality worldwide for 36 cancers in 185 countries. CA: a cancer journal for clinicians. 2018; 68:7-30
2. Postow MA, Callahan MK, Wolchok JD. Immune Checkpoint Blockade in Cancer Therapy. Journal of clinical oncology : official journal of the American Society of Clinical Oncology. 2015; 33: 1974-82

3. Topalian SL, Hodi FS, Brahmer JR, Gettinger SN, Smith DC, McDermott DF, et al. Safety, activity, and immune correlates of anti-PD-1 antibody in cancer. The New England journal of medicine. 2012; 366: 2443-54.

4. Chae YK, Pan A, Davis AA, Raparia K, Mohindra NA, Matsangou M, et al. Biomarkers for PD-1/PD-L1 Blockade Therapy in Non-Small-cell Lung Cancer: Is PD-L1 Expression a Good Marker for Patient Selection. Clinical lung cancer. 2016; 17: 350-61.

5. Proctor MJ, McMillan DC, Morrison DS, Fletcher CD, Horgan PG, Clarke SJ. A derived neutrophil to lymphocyte ratio predicts survival in patients with cancer. British journal of cancer. 2012; 107: 695-9.

6. Shimada $\mathrm{H}$, Takiguchi N, Kainuma O, Soda $\mathrm{H}$, Ikeda A, Cho A, et al. High preoperative neutrophil-lymphocyte ratio predicts poor survival in patients with gastric cancer. Gastric cancer : official journal of the International Gastric Cancer Association and the Japanese Gastric Cancer Association. 2010; 13: $170-6$

7. Kim HS, Han KH, Chung HH, Kim JW, Park NH, Song YS, et al. Neutrophil to lymphocyte ratio for preoperative diagnosis of uterine sarcomas: a case-matched comparison. European journal of surgical oncology : the journal of the European Society of Surgical Oncology and the British Association of Surgical Oncology. 2010; 36: 691-8.

8. Tomita M, Shimizu T, Ayabe T, Yonei A, Onitsuka T. Preoperative neutrophil to lymphocyte ratio as a prognostic predictor after curative resection for non-small cell lung cancer. Anticancer research. 2011; 31: 2995-8.

9. Pinato DJ, Shiner RJ, Seckl MJ, Stebbing J, Sharma R, Mauri FA. Prognostic performance of inflammation-based prognostic indices in primary operable non-small cell lung cancer. British journal of cancer. 2014; 110: 1930-5.

10. Templeton AJ, McNamara MG, Šeruga B, Vera-Badillo FE, Aneja P, Ocaña A, et al. Prognostic role of neutrophil-to-lymphocyte ratio in solid tumors: a systematic review and meta-analysis. Journal of the National Cancer Institute. 2014; 106: dju124.

11. Guthrie GJ, Charles KA, Roxburgh CS, Horgan PG, McMillan DC, Clarke SJ. The systemic inflammation-based neutrophil-lymphocyte ratio: experience in patients with cancer. Critical reviews in oncology/hematology. 2013; 88: 218-30.

12. Kang MH, Go SI, Song HN, Lee A, Kim SH, Kang JH, et al. The prognostic impact of the neutrophil-to-lymphocyte ratio in patients with small-cell lung cancer. British journal of cancer. 2014; 111: 452-60.

13. Bagley SJ, Kothari S, Aggarwal C, Bauml JM, Alley EW, Evans TL, et al. Pretreatment neutrophil-to-lymphocyte ratio as a marker of outcomes in nivolumab-treated patients with advanced non-small-cell lung cancer. Lung cancer : journal of the International Association for the Study of Lung Cancer. 2017: 106: $1-7$.

14. Diem S, Schmid S, Krapf M, Flatz L, Born D, Jochum W, et al. Neutrophil-to-Lymphocyte ratio (NLR) and Platelet-to-Lymphocyte ratio (PLR) as prognostic markers in patients with non-small cell lung cancer (NSCLC) treated with nivolumab. Lung cancer : journal of the International Association for the Study of Lung Cancer. 2017; 111: 176-81.

15. Sebastian N, Wu T, Bazan J, Driscoll E, Willers H, Yegya-Raman N, et al. Pre-treatment neutrophil-lymphocyte ratio is associated with overall mortality in localized non-small cell lung cancer treated with stereotactic body radiotherapy. Radiotherapy and oncology : journal of the European Society for Therapeutic Radiology and Oncology. 2019; 134: 151-7.

16. Huang W, Wang S, Zhang H, Zhang B, Wang C. Prognostic significance of combined fibrinogen concentration and neutrophil-to-lymphocyte ratio in patients with resectable non-small cell lung cancer. Cancer biology \& medicine. 2018; 15: 88-96.

17. Wang J, Kalhor N, Hu J, Wang B, Chu H, Zhang B, et al. Pretreatment Neutrophil to Lymphocyte Ratio Is Associated with Poor Survival in Patients with Stage I-III Non-Small Cell Lung Cancer. PloS one. 2016; 11: e0163397.

18. Hu P, Shen H, Wang G, Zhang P, Liu Q, Du J. Prognostic significance of systemic inflammation-based lymphocyte- monocyte ratio in patients with lung cancer: based on a large cohort study. PloS one. 2014; 9: e108062.

19. Cao L, Wang X, Li S, Zhi Q, Wang Y, Wang L, et al. PD-L1 is a Prognostic Biomarker in Resected NSCLC Patients with Moderate/high Smoking History and Elevated Serum SCCA Level. Journal of Cancer. 2017; 8: 3251-60.

20. Wu S, Shi X, Sun J, Liu Y, Luo Y, Liang Z, et al. The significance of programmed cell death ligand 1 expression in resected lung adenocarcinoma. Oncotarget. 2017; 8: 16421-9.

21. Takada K, Toyokawa G, Okamoto T, Shimokawa M, Kozuma Y, Matsubara T, et al. A Comprehensive Analysis of Programmed Cell Death Ligand-1 Expression With the Clone SP142 Antibody in Non-Small-Cell Lung Cancer Patients. Clinical lung cancer. 2017; 18: 572-82.e1.

22. Mori S, Motoi N, Ninomiya H, Matsuura $Y$, Nakao M, Mun M, et al. High expression of programmed cell death 1 ligand 1 in lung adenocarcinoma is a poor prognostic factor particularly in smokers and wild-type epidermal growth-factor receptor cases. Pathology international. 2017; 67: 37-44.

23. Takada K, Okamoto T, Shoji F, Shimokawa M, Akamine T, Takamori S, et al. Clinical Significance of PD-L1 Protein Expression in Surgically Resected Primary Lung Adenocarcinoma. Journal of thoracic oncology : official publication of the International Association for the Study of Lung Cancer. 2016; 11: 1879-90. 
24. Azuma K, Ota K, Kawahara A, Hattori S, Iwama E, Harada T, et al. Association of PD-L1 overexpression with activating EGFR mutations in surgically resected nonsmall-cell lung cancer. Annals of oncology : official journal of the European Society for Medical Oncology. 2014; 25: 1935-40.

25. D'Incecco A, Andreozzi M, Ludovini V, Rossi E, Capodanno A, Landi L, et al. PD-1 and PD-L1 expression in molecularly selected non-small-cell lung cancer patients. British journal of cancer. 2015; 112: 95-102.

26. Okita R, Maeda A, Shimizu K, Nojima Y, Saisho S, Nakata M. PD-L1 overexpression is partially regulated by EGFR/HER2 signaling and associated with poor prognosis in patients with non-small-cell lung cancer. Cancer immunology, immunotherapy : CII. 2017; 66: 865-76.

27. Shimoji M, Shimizu S, Sato K, Suda K, Kobayashi Y, Tomizawa K, et al. Clinical and pathologic features of lung cancer expressing programmed cell death ligand 1 (PD-L1). Lung cancer : journal of the International Association for the Study of Lung Cancer. 2016; 98: 69-75.

28. Cooper WA, Tran T, Vilain RE, Madore J, Selinger CI, Kohonen-Corish M, et al. PD-L1 expression is a favorable prognostic factor in early stage non-small cell carcinoma. Lung cancer : journal of the International Association for the Study of Lung Cancer. 2015; 89: 181-8.

29. Ameratunga M, Asadi K, Lin X, Walkiewicz M, Murone C, Knight S, et al. PD-L1 and Tumor Infiltrating Lymphocytes as Prognostic Markers in Resected NSCLC. PloS one. 2016; 11: e0153954.

30. Parsa AT, Waldron JS, Panner A, Crane CA, Parney IF, Barry JJ, et al. Loss of tumor suppressor PTEN function increases B7-H1 expression and immunoresistance in glioma. Nature medicine. 2007; 13: 84-8.

31. Green MR, Monti S, Rodig SJ, Juszczynski P, Currie T, O'Donnell E, et al. Integrative analysis reveals selective 9p24.1 amplification, increased PD-1 ligand expression, and further induction via JAK2 in nodular sclerosing Hodgkin lymphoma and primary mediastinal large B-cell lymphoma. Blood. 2010; 116: 3268-77.

32. Berry S, Taube JM. Innate vs. Adaptive: PD-L1-mediated immune resistance by melanoma. Oncoimmunology. 2015; 4: e1029704.

33. Kerr KM, Nicolson MC. Non-Small Cell Lung Cancer, PD-L1, and the Pathologist. Archives of pathology \& laboratory medicine. 2016; 140: 249-54.

34. Garon EB, Rizvi NA, Hui R, Leighl N, Balmanoukian AS, Eder JP, et al. Pembrolizumab for the treatment of non-small-cell lung cancer. The New England journal of medicine. 2015; 372: 2018-28.

35. Borghaei H, Paz-Ares L, Horn L, Spigel DR, Steins M, Ready NE, et al. Nivolumab versus Docetaxel in Advanced Nonsquamous Non-Small-Cell Lung Cancer. The New England journal of medicine. 2015; 373: 1627-39.

36. Fehrenbacher L, Spira A, Ballinger M, Kowanetz M, Vansteenkiste J, Mazieres $\mathrm{J}$, et al. Atezolizumab versus docetaxel for patients with previously treated non-small-cell lung cancer (POPLAR): a multicentre, open-label, phase 2 randomised controlled trial. Lancet. 2016; 387: 1837-46.

37. Antonia S, Goldberg SB, Balmanoukian A, Chaft JE, Sanborn RE, Gupta A, et al. Safety and antitumour activity of durvalumab plus tremelimumab in non-small cell lung cancer: a multicentre, phase $1 \mathrm{~b}$ study. The Lancet. Oncology. 2016; 17: 299-308.

38. Gaule P, Smithy JW, Toki M, Rehman J, Patell-Socha F, Cougot D, et al. A Quantitative Comparison of Antibodies to Programmed Cell Death 1 Ligand 1. JAMA oncology. 2017; 3: 256-9.

39. Ma J, Li J, Qian M, Han W, Tian M, Li Z, et al. PD-L1 expression and the prognostic significance in gastric cancer: a retrospective comparison of three PD-L1 antibody clones (SP142, 28-8 and E1L3N). Diagnostic pathology. 2018; 13: 91.

40. Parra ER, Villalobos P, Mino B, Rodriguez-Canales J. Comparison of Different Antibody Clones for Immunohistochemistry Detection of Programmed Cell Death Ligand 1 (PD-L1) on Non-Small Cell Lung Carcinoma. Applied immunohistochemistry \& molecular morphology : AIMM. 2018; 26: 83-93.

41. Scheel AH, Dietel M, Heukamp LC, Jöhrens K, Kirchner T, Reu S, et al. Harmonized PD-L1 immunohistochemistry for pulmonary squamous-cell and adenocarcinomas. Modern pathology : an official journal of the United States and Canadian Academy of Pathology, Inc. 2016; 29: 1165-72.

42. Hirsch FR, McElhinny A, Stanforth D, Ranger-Moore J, Jansson M, Kulangara $\mathrm{K}$, et al. PD-L1 Immunohistochemistry Assays for Lung Cancer: Results from Phase 1 of the Blueprint PD-L1 IHC Assay Comparison Project. Journal of thoracic oncology : official publication of the International Association for the Study of Lung Cancer. 2017; 12: 208-22.

43. Rimm DL, Han G, Taube JM, Yi ES, Bridge JA, Flieder DB, et al. A Prospective, Multi-institutional, Pathologist-Based Assessment of 4 Immunohistochemistry Assays for PD-L1 Expression in Non-Small Cell Lung Cancer. JAMA oncology. 2017; 3: 1051-8. 